\title{
ANAP Brasil
}

ISSN 1904-3240

v. 8, n. 13

\section{O USO DE AGROTÓXICOS E OS IMPACTOS NOS ECOSSISTEMAS AQUÁTICOS}

\author{
Juliana Heloisa Pinê Américo' \\ Letícia de Oliveira Manoel ${ }^{2}$ \\ Nádia Hortense Torres ${ }^{3}$ \\ Luiz Fernando Romanholo Ferreira ${ }^{4}$
}

RESUMO: A produção agrícola baseia-se na utilização intensiva de produtos químicos com a finalidade de se obter maiores valores de produtividade através do controle de pragas que afetam as culturas. No entanto, estas moléculas podem contaminar os ambientes aquáticos e atingir organismos de diferentes níveis tróficos. O presente trabalho teve como objetivo realizar uma revisão bibliográfica ressaltando os principais aspectos associados à dinâmica e destino dos agrotóxicos no ambiente bem como a contaminação dos ecossistemas aquáticos e seres vivos por estes produtos químicos. De acordo com esta pesquisa, a utilização de agrotóxicos é necessária para atender a demanda de alimentos, mas quando estes compostos atingem 0 ambiente podem persistir por muito tempo e provocar impactos nos ecossistemas aquáticos. Estes impactos envolvem alterações desde níveis celulares até mudanças populacionais e na biodiversidade aquática. A qualidade da aplicação, a escolha certa do produto e da dosagem adequada para determinada cultura além do conhecimento técnico por parte dos profissionais da área de agronomia são fatores determinantes para a manutenção da qualidade e saúde ambiental.

PALAVRAS-CHAVE: Água. Contaminação. Peixes. Inseticidas.

\footnotetext{
${ }^{1}$ Doutora em Aquicultura do Centro de Aquicultura da UNESP, Jaboticabal - SP. E-mail: americo.ju@gmail.com

${ }^{2}$ Mestre em Engenharia Civil - Recursos Hídricos e Tecnologias Ambientais, Faculdade de Engenharia de llha Solteira (FEIS/UNESP). E-mail: leticia.is@gmail.com

${ }^{3}$ Pós-doutoranda, Laboratório de Tratamento de Resíduos e Efluentes, Universidade

Tiradentes (Unit), Aracajú - SE. E-mail: nadiahortense@gmail.com

${ }^{4}$ Professor Doutor, Universidade Tiradentes (Unit), Aracajú - SE. E-mail: romanholobio@gmail.com
} 


\section{ANAP

\section{PESTICIDE USE AND IMPACTS ON AQUATIC ECOSYSTEM}

ABSTRACT: Agricultural production is based on intensive use of chemicals in order to obtain greatest yields by controlling pests that affects crops. However, these molecules can contaminate aquatic environments and achieve organisms on different trophic levels. This study aimed to conduct a literature review highlighting the main aspects related to the dynamics and fate of pesticides in environment and contamination of aquatic ecosystems and organisms by these chemicals. According to this research, the use of pesticides is necessary to meet the demand for food, because when these compounds reach the environment may persist for a long time and cause impacts on aquatic ecosystems. These impacts involve changes since cellular levels to population change and aquatic biodiversity. The application quality, the right choice of product and the proper dosage for a particular culture beyond technical knowledge on the part of agronomy professionals are key factors for maintaining quality and environmental health.

KEYWORDS: Water. Contamination. Fish. Insecticides.

\section{EL USO DE PESTICIDAS Y IMPACTOS EM ACUÁTICOS ECOSISTEMAS}

RESUMEN: La producción agrícola se basa en el uso intensivo de productos químicos con el fin de obtener información más grandes rendimientos en control de plagas que afectan a los cultivos. Sin embargo, estas moléculas pueden contaminar ambientes acuáticos y alcanzar organismos de diferentes niveles tróficos. Este estudio tiene como objetivo realizar una revisión de la literatura destacando los principales aspectos relacionados con la dinámica y el destino de los plaguicidas en el medio ambiente y la contaminación de los ecosistemas acuáticos y los seres vivos por estos productos químicos. De acuerdo a esta investigación, el uso de plaguicidas es necesario para satisfacer la demanda de alimentos, pero cuando estos compuestos alcanzan el medio ambiente puede persistir durante mucho tiempo y causar impactos en los ecosistemas acuáticos. Estos impactos incluyen cambios desde los niveles celulares a cambio de la población y la biodiversidad acuática. La calidad de las aplicaciones, la elección correcta de producto y la dosis adecuada para una cultura particular, más allá del conocimiento técnico por parte de los profesionales de la agronomía son factores clave para el mantenimiento de la calidad y la salud ambiental.

PALABRAS-CLAVE: Agua. Contaminación. Peces. Insecticidas. 


\section{ANAP $B_{r a s i}$ \\ ISSN 1904-3240 \\ v. 8, n. 13}

\section{INTRODUÇÃO}

A produção agrícola baseia-se atualmente na utilização intensiva de produtos químicos com a finalidade de se obter maiores valores de produtividade através do controle de pragas que afetam as culturas. $\mathrm{O}$ uso destes produtos químicos denominados de agrotóxicos é visto como parte fundamental no sucesso da cadeia produtiva agrícola.

Segundo o Sindicato Nacional da Indústria de Produtos para Defesa Agrícola (SINDAG), apesar do expressivo crescimento da área cultivada com sementes transgênicas; tecnologia que poderia substituir e reduzir o uso do controle químico, constata-se que as vendas dos agrotóxicos no país aumentaram mais de $72 \%$ entre 2006 e 2012 - de 480,1 mil para 826,7 mil toneladas. As vendas de inseticidas aumentaram quase $84 \%$; de 93,1 mil para 170,9 mil toneladas e as de herbicida, 44\%; 279,2 mil toneladas em 2006 para 403,6 mil toneladas em 2012. As vendas de agrotóxicos foram de quase US\$ 8,5 bilhões em 2011, o dobro do ocorrido em 2005. Com estas vendas e uso de agrotóxicos, o Brasil destaca-se com o segundo maior mercado mundial; fica atrás apenas dos Estados Unidos (SINDAG, 2012).

Os agrotóxicos são moléculas sintetizadas para afetar determinadas reações bioquímicas de microrganismos, animais e plantas que se deseja controlar ou eliminar. No entanto, existem processos bioquímicos comuns entre alguns seres vivos e, dessa forma, o efeito pode atingir além dos organismos alvos outros seres vivos não alvos presentes no ambiente (SPADOTTO et al., 2004).

Originalmente, os defensivos agrícolas possuíam baixa mobilidade e solubilidade e tinham um forte poder de adesão ao solo, no entanto com a evolução tecnológica, estes produtos tornaram-se mais solúveis em água, com baixa capacidade de adesão e mais voláteis. Essas inovações tecnológicas que se baseavam na manipulação de compostos químicos criaram agrotóxicos 


\section{ANAP $B_{r a s i l}$ \\ ISSN 1904-3240 \\ v. 8, n. 13}

\section{REVISTA CIENTIFICA 2015}

cada vez mais tóxicos, persistentes e eficientes pra combater pragas (VEIGA et al., 2006).

O aporte dos agroquímicos nos ecossistemas aquáticos a partir do uso agrícola depende, em grande parte, da dinâmica destes compostos no solo, uma vez que, além do carreamento pela ação dos ventos com posterior precipitação, a movimentação dos agrotóxicos a partir do solo contribui de forma significativa para sua entrada no meio aquático (ISENSEE, 1991).

Os agrotóxicos presentes em corpos d'água podem penetrar nos organismos aquáticos através de diversas portas de entrada e seu grau de acumulação depende do tipo de cadeia alimentar, da disponibilidade e persistência do contaminante na água e especialmente de suas características físicas e químicas (SPACIE ; HAMELINK, 1985).

O presente trabalho teve como objetivo realizar uma revisão bibliográfica ressaltando os principais aspectos relacionados à dinâmica e destino dos agrotóxicos no ambiente bem como a contaminação dos ecossistemas aquáticos e seres vivos devido ao uso destes produtos químicos.

\section{DESENVOLVIMENTO}

\section{Dinâmica e destino ambiental dos agrotóxicos}

A partir da aplicação de um agrotóxico seja ela via sólida, líquida ou gasosa vários processos físicos, químicos, físico-químicos e biológicos podem influenciar no seu comportamento no ambiente. O destino destes produtos é governado por processos de retenção (adsorção, absorção e sorção), de transformação (degradação biótica e abiótica), de transporte (deriva, volatilização, lixiviação e carreamento superficial), e por interação desses processos (SPADOTTO, 2006).

O solo é o destino final dos produtos químicos usados na agricultura, sejam eles aplicados diretamente neste ou sob a parte aérea das plantas. Ao 


\section{ANAP $B_{\text {rasil }}$ \\ ISSN 1904-3240 \\ v. 8, n. 13}

REVISTA

entrarem em contato com o solo, os agrotóxicos estão sujeitos à sorção, que significa a retenção da molécula do agrotóxico com o solo. Em alguns casos menos de $0,1 \%$ dos agrotóxicos alcançam o alvo, enquanto que o restante $(99,9 \%)$ tem potencial para se mover para outros compartimentos ambientais, como os mananciais hídricos (SABIK; JEANNOT; RONDEAU, 2000).

Segundo Regitano (2002), a capacidade de sorção de um agrotóxico ao solo é influenciada por diferentes propriedades da molécula como a solubilidade em água, a constante de ionização ácido-base, a pressão de vapor e o coeficiente de partição octanol-água, além das propriedades físicas e químicas do solo. As constantes de ionização ácido-base assumem papel importante no comportamento dos herbicidas sendo que a grande maioria dos fungicidas, inseticidas e acaricidas não é ionizável.

No processo de absorção, o agrotóxico penetra em um ou mais organismos através de sua membrana celular e assim sofre alterações enquanto que na adsorção o composto fica aderido à superfície das partículas e pode sofrer influências da solução do solo (SPADOTTO et al., 2010).

A volatilização dos agrotóxicos também é um importante meio de distribuição desses produtos no ambiente, pois possibilita o carreamento dos compostos através da atmosfera e, posteriormente, sua deposição nas águas superficiais através da precipitação (SILVA FILHO, 2009).

De acordo com Spadotto et al. (2010), a volatilização pode ocorrer durante e após a aplicação, a partir da superfície das plantas, na superfície e na matriz do solo, assim como na superfície e na coluna d'água. Além disso, as estimativas de resíduos têm de considerar também os processos de transporte na atmosfera e a deposição no solo, na vegetação e em corpos d'água. Segundo Carter (2000) as perdas de herbicidas por volatilização podem variar de menos de $2 \%$ até $90 \%$.

Os agrotóxicos que são lixiviados são considerados potenciais poluidores das águas subterrâneas. A extensão da contaminação desses ambientes aquáticos depende das propriedades dos agrotóxicos, das 


\section{ANAP $B_{\text {rasil }}$ \\ ISSN 1904-3240 \\ v. 8, n. 13}

\section{REVISTA C IENTIFICA}

características do solo, da velocidade de drenagem e da profundidade do lençol freáticos. O uso do solo, as condições hidrogeológicas do local e o grupo geológico do solo são outros fatores que também podem afetar a concentração dos agrotóxicos nas águas subterrâneas (MULLER et al., 1995).

O carreamento superficial corresponde ao movimento do agrotóxico pela água de enxurrada na superfície do solo, podendo chegar à superfície dos rios, lagos, córregos, açudes e terrenos com declividade baixa (SILVA FILHO, 2009). Segundo Spadotto et al. (2010), o carreamento superficial é uma importante rota de deslocamento dos agrotóxicos, pois em seus momentos iniciais após a aplicação o composto pode ser carreado para fora da área em solução na água de escoamento ou, ainda pode ser adsorvido fortemente às argilas e à matéria orgânica sendo transportado pelo arraste das partículas do solo.

Outro problema muito comum em relação à dinâmica e destino de agrotóxicos no ambiente é o processo de deriva, no qual o composto é transportado através do vento durante sua aplicação nas culturas ou no solo. A ausência de vento também pode ser prejudicial, pois as gotas muito finas podem ficar suspensas no ar devido à estabilidade atmosférica, dispersando-se por vários quilômetros do local de aplicação, sendo, muitas vezes, somente removida da atmosfera pela ação da chuva (SPADOTTO, 2006).

$\mathrm{Na}$ Tabela 1 são apresentados resumidamente os processos, as consequências e os fatores que são capazes de relocar as moléculas de agrotóxicos nos diferentes compartimentos ambientais (água, ar e solo) sem alterar sua estrutura.

Tabela 1. Processos que relocam as moléculas de agrotóxicos sem alterar sua estrutura 


\section{ANAP Brasil \\ ISSN 1904-3240 \\ v. 8, n. 13}

\section{REVISTA C I EN TIF I C A}

\begin{tabular}{|c|c|c|}
\hline Processos & Consequência & Fatores \\
\hline Deriva & Movimento pela ação do vento & $\begin{array}{l}\text { Velocidade do vento e tamanho } \\
\text { das gotas }\end{array}$ \\
\hline Volatilização & $\begin{array}{l}\text { Perda por evaporação do solo, da planta } \\
\text { ou do ecossistema aquático }\end{array}$ & $\begin{array}{c}\text { Pressão de vapor, velocidade do } \\
\text { vento e temperatura }\end{array}$ \\
\hline Adsorção & $\begin{array}{l}\text { Remoção pela interação com plantas, } \\
\text { solo e sedimento }\end{array}$ & $\begin{array}{c}\text { Conteúdo mineral e matéria } \\
\text { orgânica, tipo de mineral e } \\
\text { umidade }\end{array}$ \\
\hline $\begin{array}{c}\text { Ribas } \\
\text { Absorção }\end{array}$ & $\begin{array}{c}\text { Absorção pelas raízes ou ingestão } \\
\text { animal }\end{array}$ & $\begin{array}{l}\text { Transporte pela membrana } \\
\text { celular, tempo de contato e } \\
\text { suscetibilidade }\end{array}$ \\
\hline Lixiviação & $\begin{array}{l}\text { Translocação lateral e vertical através } \\
\text { do solo }\end{array}$ & $\begin{array}{l}\text { Conteúdo de água, macroporos, } \\
\text { textura do solo, quantidade do } \\
\text { mineral e conteúdo da matéria } \\
\text { orgânica }\end{array}$ \\
\hline Erosão & Movimento pela ação da água ou vento & $\begin{array}{l}\text { Chuva, velocidade do vento, } \\
\text { tamanho das partículas do } \\
\text { mineral e da matéria orgânica } \\
\text { com moléculas adsorvidas }\end{array}$ \\
\hline
\end{tabular}

Fonte: PIERZYNSKI; SIMS; VANCE (1994)

No ambiente, a transformação de agrotóxicos assim como de qualquer outro xenobióticos pode ocorrer através da degradação abiótica e biótica. De acordo com Zagatto e Bertoletti (2008), a degradação abiótica ocorre através de transformações químicas como fotólise, oxidação/redução, hidrólise e reações de troca iônica enquanto que a degradação biótica ou biodegradação envolve a ação de microrganismos presentes no ambiente, os quais utilizam tais substâncias diretamente como substrato, a fim de obter energia necessária ao seu crescimento e metabolismo.

A biodegradação de determinado xenobiótico depende da sua disponibilidade no ambiente para os organismos que promoverão a degradação. Muitos dos produtos mais recalcitrantes apresentam baixa degradação devida sua hidrofobicidade. Acredita-se que somente agrotóxicos 


\section{ANAP $B_{\text {rasil }}$ \\ ISSN 1904-3240 \\ v. 8, n. 13}

presentes na solução do solo estão disponíveis para a ação microbiana (MATA-SANDOVAL, KANS e TORRENTS, 2002).

Além disso, solos que contém maior teor de matéria orgânica possuem maior atividade microbiana, o que pode possibilitar uma degradação mais rápida dos agrotóxicos pela ação dos microrganismos (SILVA FILHO, 2009). De acordo com estudo de Rocha et al. (2013), a persistência do herbicida diuron é dependente dos atributos físicos e químicos dos solos, sendo a sua dissipação menor em solos com maior teor de matéria orgânica. A correção do $\mathrm{pH}$ do solo favorece a sua degradação apenas em solos arenosos com baixo teor de matéria orgânica.

$\mathrm{Na}$ Tabela 2 são apresentados resumidamente os processos que são capazes de alterar a estrutura química dos agrotóxicos no ambiente.

Tabela 2. Processos que alteram a estrutura química dos agrotóxicos no ambiente

\begin{tabular}{|c|c|c|}
\hline Processos & Consequência & Fatores \\
\hline Fotoquímica & $\begin{array}{l}\text { Quebra da molécula devido a absorção } \\
\text { de luz solar }\end{array}$ & $\begin{array}{l}\text { Estrutura química, intensidade e } \\
\text { duração da luz solar e exposição }\end{array}$ \\
\hline Microbiana & Degradação por microrganismos & $\begin{array}{l}\text { pH, umidade, temperatura, } \\
\text { condições dos nutrientes, } \\
\text { conteúdo da matéria orgânica }\end{array}$ \\
\hline Química & $\begin{array}{c}\text { Alteração por processos químicos como } \\
\text { hidrólise e reações de oxi-redução }\end{array}$ & $\mathrm{pH}$ e fatores ambientais \\
\hline Metabolismo & $\begin{array}{c}\text { Transformação química após absorção } \\
\text { pelas plantas e animais }\end{array}$ & $\begin{array}{c}\text { Capacidade de ser absorvido, ser } \\
\text { metabolizado e interagir com } \\
\text { organismos }\end{array}$ \\
\hline
\end{tabular}

Fonte: PIERZYNSKI; SIMS; VANCE (1994)

\section{Ocorrência da contaminação de ecossistemas aquáticos por agrotóxicos}

Diferentes estudos relatam a presença de resíduos de agrotóxicos (herbicidas, inseticidas, nematicidas e fungicidas) em rios, córregos e águas subterrâneas em vários países (TANABE et al., 2000; CEREJEIRA et al., 2003; 


\section{ANAP $B_{r a s i l}$ \\ ISSN 1904-3240 \\ v. 8, n. 13}

REVISTA C I EN TIF I C A

RODRIGUEZ-MOZAZ et al., 2004; GRÜTZMACHER et al., 2008; TANG et al., 2013).

Marchezan et al. (2003) ao estudarem a dispersão de herbicidas em águas da Bacia Hidrográfica dos Rios Vacacaí e Vacacaí-Mirim no Sul do Brasil registraram a presença de clomazone, quinclorac e propanil em 18 amostras das 45 coletadas.

Resíduos de carbofuran, quinclorac, clomaze e fipronil foram encontrados nas águas do Canal São Gonçalo e do Rio Piratini na região Sul do Brasil em áreas de cultivo de arroz irrigado. Entre os agrotóxicos detectados o carbofuran e o quinclorac foram os mais persistentes (GRÜTZMACHER et al., 2008).

Inseticidas organofosforados e carbamatos foram encontrados em amostras de água superficial no município de Paty do Alferes que é o maior produtor de tomate do Estado do Rio de Janeiro (VEIGA et al., 2006). Tang et al. (2013) detectaram os pesticidas diclorodifeniltricloroetano (DDT), endosulfan, clordano e aldrin no Rio Yangtze (China).

No Rio Shimano (Japão), Tanabe et al. (2000) relataram a ocorrência do herbicida simazina enquanto que no Rio Llobregat localizado no noroeste da Catalunha (Espanha) Rodriguez-Mozaz et al. (2004) detectaram a presença de outros herbicidas (atrazina, diuron) além da simazina.

Os pesticidas alacloro, metolacloro, metribuzim, simazina e atrazina foram detectados no período de 1991 a 1998 em águas subterrâneas localizadas em sete áreas agrícolas em Portugal, sendo que os herbicidas encontrados com maior frequência foram atrazina, simazine e alacloro (CEREJEIRA et al., 2003).

Todos os relatos a respeito da presença de resíduos de agrotóxicos em ambientes aquáticos estão relacionados com o alto nível de industrialização, a necessidade do aumento da produção de alimentos juntamente com a alta densidade populacional, que estão distribuídas principalmente em regiões 


\section{ANAP $B_{r a s i l}$ \\ ISSN 1904-3240 \\ v. 8, n. 13}

\section{REVISTA CIENTIFICA 2015}

geográficas próximas as partes mais baixas dos rios (ZAGATTO; BERTOLETTI, 2008).

\section{Impactos dos agrotóxicos nos ecossistemas aquáticos}

O aumento no volume de agrotóxicos aplicados tem trazido uma série de transtornos e modificações para o ambiente, tanto pela contaminação das comunidades de seres vivos que o compõe, quanto pela sua acumulação nos segmentos bióticos e abióticos dos ecossistemas (biota, água, ar, solo, etc.) Um dos efeitos indesejáveis provocado pelos agrotóxicos é a contaminação de organismos não alvos, ou seja, espécies que não interferem no processo de produção (RIBAS; MATSUMURA, 2009).

De acordo com Zagatto e Bertoletti (2008), a assimilação e a retenção destes contaminantes por um organismo podem acarretar a concentração elevada do mesmo, com probabilidade de casar efeitos deletérios. Esse processo pode ocorrer por absorção direta, a partir do ambiente, ou por absorção indireta, através do alimento contaminado. Consequentemente, em uma cadeia alimentar, os contaminantes podem ser transferidos de um nível trófico a outro.

Os efeitos dos agrotóxicos podem ser letais ou subletais e são observados em organismos aquáticos pertencentes a diferentes níveis tróficos como algas e macrófitas (produtores), microcrustáceos e moluscos (consumidores primários), e peixes (consumidores secundários e terceários).

Os efeitos letais ou agudos envolvem respostas que interferem nos processos celulares intensamente, causando a morte rapidamente. Muitas vezes, podem se expressar através de respostas que antecedem a morte, como sufocamento e interferências nos movimentos dificultando a fuga de predadores ou a obtenção de alimento (AZEVEDO; CHASIN, 2003).

Segundo Azevedo e Chasin (2003), os efeitos subletais provocam alterações nas atividades fisiológicas e comportamentais, sem mortalidade 


\section{ANAP $B_{\text {rasil }}$ \\ ISSN 1904-3240 \\ v. 8, n. 13}

imediata. Esta pode ocorres ao longo do tempo devido a interferências na alimentação, crescimento e sistema imunológico, estes efeitos causam mudanças populacionais ou na composição e diversidade de espécies nos ambientes aquáticos.

Muitos estudos avaliaram a toxicidade aguda (efeito letal) de agrotóxicos em diferentes espécies de microcustáceos, peixes e anfíbios, mostrando que baixas concentrações destes contaminantes em água podem provocar a morte destes organismos (FRELLO, 1998; RESGALLA JUNIOR et al., 2002; NAKAGOME; NOLDIN; RESGALLA, 2007; FRANÇA-SALGUEIRO, 2013; MANSANO;MOREIRA;ROCHA, 2013). Os valores de toxicidade aguda são baseados na concentração do pesticida que causa mortalidade para $50 \%$ dos organismos expostos.

$\mathrm{Na}$ tabela 3 encontram-se valores de toxicidade aguda do inseticida carbofuran (muito utilizado na cultura de cana de açúcar) microscrustáceo, peixe e anfíbio.

Tabela 3. Toxicidade aguda baseada nos valores de $\mathrm{CE}_{50} / \mathrm{CL}_{50}$ para diferentes organismos aquáticos expostos ao inseticida carbofuran

\begin{tabular}{ccc}
\hline Organismos & $\mathbf{C E}_{50} / \mathrm{CL}_{50}$ & Referência \\
\hline Ceriodaphnia silvestrii & $0,75 \mu \mathrm{g} \cdot \mathrm{L}^{-1}$ & Mansano;Moreira; Rocha (2013) \\
Poecilia reticulata & $0,165 \mathrm{mg} \cdot \mathrm{L}^{-1}$ & Frello (1998) \\
Danio rerio & $1,34 \mathrm{mg} \cdot \mathrm{L}^{-1}$ & Nakagome; Noldin; Resgalla Junior (2007) \\
Lithobates catesbeianus & $29,90 \mathrm{mg} \cdot \mathrm{L}^{-1}$ & França-Salgueiro (2013) \\
\hline
\end{tabular}

No caso dos efeitos subletais, os agrotóxicos podem acumular-se em tecidos, alterar funções celulares, causar lesões histológicas, alterar comportamento de peixes afetando diretamente o sistema nervoso, ou indiretamente, afetando outras funções orgânicas como osmorregulação e metabolismo de hormônios sexuais. A exposição crônica a estes contaminantes pode afetar níveis organizacionais mais superiores, como por 


\section{ANAP Brasil \\ ISSN 1904-3240 \\ v. 8, n. 13}

\section{REVISTA C I ENTIFICA}

exemplo, pode deprimir o crescimento e o processo reprodutivo, que é um dos processos mais afetados pela poluição. Finalmente, alterações na função de um grupo de organismos em um ecossistema causam efeitos em outros grupos que sejam suas presas ou predadores (HEATH, 1995). Na tabela 4 pode ser observada a relação inversa entre a solubilidade aquosa dos agrotóxicos e sua acumulação em peixes.

Tabela 4. Relação inversa entre a solubilidade aquosa dos agrotóxicos e sua acumulação em peixes

\begin{tabular}{ccc}
\hline Composto & Solubilidade aquosa (ppm) & $\begin{array}{c}\text { Acumulação máxima (peixe } \\
\text { inteiro) }\end{array}$ \\
\hline Lindane & 10 & $100 \mathrm{X}$ \\
Toxafeno & 3 & $10.000 \mathrm{X}$ \\
Dieldrin & 0,25 & $10.000 \mathrm{X}$ \\
DDT & $0,0012-0,037$ & 100.000 a $1.000 .000 \mathrm{X}$ \\
2,4 D & 725 & $150 \mathrm{X}$ \\
\hline
\end{tabular}

Fonte: ZAGATTO; BERTOLETTI (2008)

Embora o uso de muitos pesticidas como os organoclorados esteja proibido para determinados fins, ainda estão presentes no ambiente, podendo causar impacto significativo sobre varias populações, até mesmo de aves aquáticas (ZAGATTO; BERTOLETTI, 2008).

\section{CONSIDERAÇÕES FINAIS}

Atualmente a utilização de agrotóxicos é necessária para a manutenção do sucesso da cadeia produtiva agrícola e para atender a demanda de alimentos. No entanto, quando estes compostos atingem o ambiente podem persistir por muito tempo e provocar impactos nos ecossistemas aquáticos. Estes impactos envolvem alterações desde níveis celulares nos organismos até mudanças populacionais e na biodiversidade aquática. 


\section{ANAP

Os impactos causados pelo uso de agrotóxicos podem ser minimizados por meio da utilização consciente destes produtos químicos. A qualidade da aplicação, a escolha certa do produto e da dosagem adequada para determinada cultura além do conhecimento técnico por parte dos profissionais da área de agronomia são fatores determinantes para a manutenção da qualidade e saúde ambiental.

\section{REFERÊNCIAS}

AZEVEDO, F. A.; CHASIN, A. A. M. As bases toxicológicas da ecotoxicologia. São Carlos: RiMa, São Paulo: Intertox, 2003, 340p.

CARTER, A. D. Herbicide movement in soils: principles, pathways and processes. Weed Research, v. 40; p. 113-122, 2000

CEREJEIRA, M. J.; VIANA, P.; BATISTA, S.; PEREIRA, T.; SILVA, E.; VALÉRIO, M. J.; SILVA, A.; FERREIRA, M.; SILVA-FERANDES, A. M. Pesticides in Portuguese surface and ground waters. Water Research, v. 37, n. 5, p. 1055-1063, 2003.

FRANÇA-SALGUEIRO, F.M. Avaliação da toxicidade de agrotóxicos utilizados na cultura do arroz irrigado para girinos de Lithobates catesbeianus. 2013. 101p. Tese (Doutorado em Ciência) - Universidade de São Paulo.

FRELLO, C.P. Avaliação da toxicidade aguda do agrotóxico carbofuran utilizando reativos biológicos: Poecila reticulata e Daphnia magna. 1998. 96p. Dissertação (Mestrado em Engenharia Ambiental) - Universidade Federal de Santa Catarina.

GRÜTZMACHER, D. D.; GRÜTZMACHER, A. D.; AGOSTINETTO, D.; LOECK, A. E.; ROMAN, R.; PEIXOTO, S. C.; ZANELLA, R. Monitoramento de agrotóxicos em dois mananciais hídricos no sul do Brasil. Revista Brasileira de Engenharia Agrícola e Ambiental, v.12, n.6, p. 632637, 2008.

HEATH, A.G. Water pollution and fish physiology. $2^{a}$ ed. Lewis Publishers, Boca Raton.1995.

ISENSEE, A. R. Movement of herbicides in terrestrial and aquatic environments. In: PIMENTEL, D. CRC Handbook of pest management in agriculture. Boca Raton: CRC Press, 1991. v. 1, p. 651-659.

MANSANO, A. S.; MOREIRA, R. A.; ROCHA, O. Toxicidade aguda do agrotóxico carbofuran ao cladócero Ceriodaphnia silvestrii daday. Fórum Ambiental da Alta Paulista, v.9, n.11, p.91103, 2013.

MARCHEZAN, E.; SANTOS, F. M. DOS; CAMARGO, E. R.; MACHADO, S. L. DE O.; ZANELLA, R.; ÁVILA, L. A. DE; GONÇALVES, F. F.; PRIMEL, E. G.; MACEDO, V. R. M. Monitoramento em rios e persistência de herbicidas em arroz irrigado. Santa Maria: UFSM, 2005. 8p. Informe Técnico, 05. 


\section{ANAP

MATA-SANDOVAL, T.; KANS, J.; TORRENTS, A. Infl uence of rhamnolipids and triton X-100 on the desorption of pesticides from soils. Environmental Science and Techology, v. 36, p. 4669-4675, 2002

MULLER, D. K.; HAMILTON, P. A.; HELSEL, D. R.; HITT, K. J.; RUDDY, B. C. Nutrients in groundwater and surface water of the United States: an analysis of data through 1992. Denver: United States Geological Survey, 1995. (Water Resource Inventory Report, 95- 4031).

NAKAGOME, F.K.; NOLDIN, J.A.; RESGALLA JUNIOR, C. Toxicidade aguda de alguns herbicidas e inseticidas utilizados em lavouras de arroz irrigado sobre o peixe Danio rerio. Pesticidas: Revista de Ecotoxicologia e Meio Ambiente, v.17, p.117-122, 2007.

PIERZYNSKI, G.M.; SIMS, J.T.; VANCE, G.F. Organic chemicals in the environment. In: PIERZYNSKI, G.M.; SIMS, J.T.; VANCE, G.F. ed. Soils and environmental quality. Boca Raton: Lewis Publishers, 1994, p. 185-215.

REGITANO, J. B. Propriedades físico-químicas dos defensivos e seu destino no ambiente. In: SIMPÓSIO SOBRE A DINÂMICA DE DEFENSIVOS AGRÍCOLAS NO SOLO - ASPECTOS PRÁTICOS E AMBIENTAIS, 2002, Piracicaba. Anais... Piracicaba: ESALQ, 2002. p. 40-50.

RESGALLA JUNIOR, C.; NOLDIN, J.A.; SANTOS, A.L.; SATO, G.; EBERHARDT, D.S. Toxicidade aguda de herbicidas e inseticidas utilizados na cultura do arroz irrigado sobre juvenis de carpa (Cyprinus carpio). Pesticidas: Revista de Ecotoxicologia e Meio Ambiente, v.12, p.59-68, 2002.

RIBAS, P. P.; MATSUMURA, A. T. S. A química dos agrotóxicos: impacto sobre a saúde e meio ambiente. Revista Liberato, v.10, n.14, p. 149-158, 2009.

ROCHA, P. R. R.; FARIA, A. T.; SILVA, G. S.; QUEIROZ, M. A. L. R.; GUIMARÃES, F. F. N.; TIRONI, S. P.; GALON, L.; SILVA, A. A. Meia vida do diuron em solos com diferentes atributos físicos e químicos. Ciência Rural, v.43, n.11, p. 1961-1966. 2013.

RODRIGUEZ-MOZAZ, S.; ALDA, M. J. L.; BARCELÓ, D. Monitoring of estrogens, pesticides and bisphenol $A$ in natural Waters and drinking water treatment plants by solid-phase extraction-liquid chromatography-mass spectrometry. Journal of Chromatography A, v. 1045, p. 85-92, 2004.

SABIK, H.; JEANNOT, R.; RONDEAU, B. Multiresidue methods using solid-phase extraction techniques for monitoring piority pesticides, including triazines and degradation products, in ground and surface waters. Journal of Chromatography, v. 885, n. 1-2, p. 217-236, 2000.

SILVA FILHO, C. F. Determinação de resíduos dos pesticidas pirimetanil, pirimicarbe e buprofezina em águas ambientais por SPME e GC-MS. 2009. 101p. Dissertação (Mestrado em Química) - Núcleo de pós-graduação em Química, Universidade Federal de Sergipe, São Cristóvão-SE, 2009.

SINDICATO NACIONAL DA INDÚSTRIA DE PRODUTOS PARA DEFESA AGRÍCOLA (SINDAG - 2012). Disponível em: < http://www.sindag.com.br/> Acesso: 20 agosto.2012.

SPACIE, A.; HAMELINK, J.L. Bioacumulation. In: RAND, G.M. \& PETROCELLI, S.R., (Ed.). Fundamentals of aquatic toxicology: methods and applications, New York: Hemisphere, 1985. p.495-525. 


\section{ANAP

SPADOTTO, C. A.; GOMES, M. A. F.; LUCHINI, L. C.; ANDRÉA, M. M. Monitoramento de risco ambiental de Agrotóxicos: princípios e recomendações. Documentos 42. EMBRAPA:CNPMA, Jaguariúna, 29p., 2004.

SPADOTTO, C. A. Avaliação de riscos ambientais de agrotóxicos em condições brasileiras. Documentos 58. EMBRAPA:CNPMA, Jaguariúna, 20p., 2006.

SPADOTTO, C. A.; SCORZA JUNIOR, R. P.; DORES, E. F. G. C.; GEBLER, L.; MORAES, D. A. C. Fundamentos e aplicações da modelagem ambiental de agrotóxicos. Documento 78. EMBRAPA:CNPMA, Jaguariúna, 46p., 2010.

TANABE, A.; MITORE, H.; KAWATA, K.; SAKAI, M. New monitoring system for ninety pesticides and related compounds in river water by solid-phase extraction with determination by gás chromatography/mass spectrometry. J. AOAC Int., v. 83, n. 1, p. 61-77, 2000.

TANG, Z.; HUANG, Q.; YANG, Y.; ZHU, X.; HAIHUI, F. Organochlorine pesticides in the lower reaches of Yangtze River: Occurrence, ecological risk and temporal trends. Ecotoxicology and Environmental Safety, v.87, p. 89-97, 2013.

VEIGA, M. M.; SILVA, D. M.; VEIGA, L. B. E; FARIA, M. V. C. Análise da contaminação dos sistemas hídricos por agrotóxicos numa pequena comunidade rural do Sudeste do Brasil.

Caderno de Saúde Pública, v.22, n.11, p.2391-2399, 2006.

ZAGATTO, P.A; BERTOLETTI, Ecotoxicologia aquática: Princípio e aplicações. $2^{a}$ ed. São Carlos. Rima, 2008. 472p. 